\title{
A Study on How to Breathe Properly When Practicing
}

\section{Tai Chi Chuan}

\author{
Hanchun Yang \\ Sports Department, Nanjing Institute of Industry Technology \\ Nanjing, 210046, China
}

Received: March 15, 2011 Accepted: March 26, 2011 doi:10.5539/hes.v1n1p64

\begin{abstract}
When practicing Tai Chi Chuan, proper breath plays an important role in shaping Tai Chi Chuan's style and its fitness value. The paper aims to analysis the postures of Tai Chi Chuan and its breath characteristics. The paper also presents some new insights on how to co-ordinate breath with postures by case studies.
\end{abstract}

Keywords: Tai Chi Chuan, Breath, Case studies

Tai Chi Chuan is a kind of boxing that both the inside and outside need to be exercised. The postures focus on the appearance, and the outer exercises which means the same as the saying "Practice outside muscles skin". There is no doubt that the inner exercises refers to breath. Normally, there are two ways of practicing breath: tuna and guidance. The practice of boxing and breathing are closely connected in Tai Chi Chuan. Breath is refers to tuna in Chi Gong. When practicing Tai Chi Chuan, breath is connected with boxing to make a series of continuous move. As a consequence, the breath methods of Tai Chi Chuan and the breath methods of Chi Gong have the same origin. It can be said that practicing boxing along with Chi Gong, using the outer force to drive the inner force. Since the outer factor exerts on the inner, both can be exercised. Hence it can make people stronger and also cure diseases. So the importance of breathing when practicing Tai Chi Chuan cannot be overstated.

This paper begins with Tai Chi Chuan's appearance and breathe characteristics and presents some new points on how to deal with the breathe issues when practicing Tai Chi Chuan based on the author's teaching experiences and extensive literatures. It will be helpful in shaping Tai Chi Chuan's style and fitness value.

\section{Requirements of Appearance When Practicing Tai Chi Chuan}

The practice of shape is the basis of Tai Chi Chuan, which emphasizes on naturalness, easiness, and tranquility. To be specific, its requirements are as follows: lower the shoulder and drop the elbows, empty the breast and fulfill the stomach, pulling the backbone, back the forechest and forward the back, fix the waist and drop the hip, aiming to achieve perfectness. The learners should be in absolute quietness and leave all distracting thoughts, and absorbedly preparing to practice Tai Chi Chuan.

The four must dos when practicing Tai Chi Chuan are lightness, slowness, roundness and evenness. Lightness means that when you move, you must be light. It is not easy for the beginners to do this. However, after gradual practices, it could be achieved. Mastering lightness is the basis of the following practices. Slowness means that the move of Tai Chi Chuan is much slower that other kind of boxing, especially in the beginning period, the learners should be intentionally slow. This will prevent the learners from moving wrongly and also helping them keep the habit of slowing down. When they are flexible in doing this, they can also keep a slow speed when practicing Tai Chi Chuan. Roundness means that every move in Tai Chi Chan should be done in circle. Even if it is a petty action, it should keep its fixed circle. In one word, the practicing of Tai Chi Chuan is done in circle. Evenness refers that when practicing, a corresponding constant speed is needed to finish the whole move. Namely, the movement should be considered as innumerable spots but not only beginning and finishing spots, however slow the motion is. It will make the move progress as spots. After long practices, the movement will not lose its evenness and it is also helpful in abstaining from the habit of unstableness.

The purpose of practicing boxing is to dredge the channel and agitate the fascia. Since the fascia is connected with the muscle and the channel is below the fascia and in the muscle, the bones stretch under the guidance of 
consciousness, so the channel can be dredged and the qi and blood can recycle. Therefore, the hypogastric can be nourished.

\section{The Breath Methods in Practicing Tai Chi Chuan}

The beginners can use the nose to inhale and use the mouth to exhale. They should breathe naturally. When they are familiar with the postures, and will not forget the postures, the learners can practice breath along with the postures.

When practicing Tai Chi Chuan, we normally adopt the deep abdominal breathing method. It has two practicing methods. One is along and the other is against. Both of them are breathing exercises that motivated by consciousness. With the diaphragm going up and down, the muscles of the breast, back and stomach loose and rotate, which facilitates the internal organs to move and enhance the blood circulation. As a consequence, metabolism within the body can be improved. Hence, it is helpful to build health and is good for cure and preventing chronic diseases.

\subsection{Along Breathing Method}

Along breathing is also called isometric breathing. Its characteristics are: the force brought by breath is strong and it can efficiently utilized, which is the reason why it is frequently used in daily practices. When adopting this method, the tip of the tongue should touch the palate and inhale with the nose, drop the diaphragm and fulfill the lower abdomen; when exhale, people should slightly open the mouth and the tip of the tongue should leave the palate, pull the lower abdomen and image that the air you have just inhaled has been sent to the lower abdomen. The whole progress should be done naturally to prevent from feeling suffocated. The along breath method is only suitable for formulary pile step practice and static practice but not suitable for dynamic boxing practice.

\subsection{Against Breathing Method}

Against breathing method is also called transfiguration breathing. When practicing this breathing method, people should use nose and mouth to breath. Using consciousness and boxing postures as a lead and the whole body should be relaxed so as to drive the air. In contrast to along breathing method, when inhale, the lower abdomen should be pulled, the diaphragm should be leveled and the lung should expand naturally; and when exhale the diaphragm should be dropped and the lung should stretch downward naturally. Open the mouth slightly and the tip of the tongue should leave the palate, imagining that passing the air gradually to the public region. When practicing the boxing postures, the learner should consciously imagine that all breathe come from the public region, which will refresh the air. The breath should be deep, long, thin, even, slow and quite.

The against breathing method is more difficult to master than the along breathing method. If the learner use the along breathing method to match with boxing practices, the air are always in the public region, which is not helpful for practicing boxing. Against breathing rule is in accordance with the rule of boxing. Since all movement needs the reaction force of the ground, only when the air dropped in the public area, can the force be put forwarded. When putting forward the force, the mouth should exhale rapidly, making the air dropped to the public area and fulfill the belly. Using the air to drive force and speak aloud to enhance its force. It is a vivid coordination of force and breath.

\section{Cases of Application of Breath in Practicing Tai Chi Chuan}

The coordination of Tai Chi Chuan and breathe is not easy. Different from Chi Gong, Tai Chi Chuan is a kind of dynamic boxing, so when practicing the boxing, the body is in various movements and the postures continuously change, the moving route of hands and feet are also different from each other. Though Chi Gong also has dynamic movements, it is comparatively easier. Its postures are repeated, so it is easy to coordinate. Now, the dynamic and the static are combined and fused. People practicing Tai Chi Chuan is expected to sweat but not pant, which is impossible for other boxing. The following is several typical postures in Tai Chi Chuan.

(i) Standing on one leg. Raise the body and the knee and inhale. Then bend the knee, change the foot and exhale. The boxing postures have raising move and descending move so when the body raise inhale and when the body descend exhale.

(ii) Part the wild horse's mane on right side. Holding the ball from the left side and inhale; then turn to right and exhale.

(iii) Forearm rollings on both sides. Inhale when turning the waist and winding the hand, applying force when putting forward the palm and exhale.

(iv) Grasp bird's tail. Inhale when changing the postures from forward stance to recoil virtual stance and exhale when changing the postures from recoil virtual stance to forward stance. 
The above four cases aims to analyze the coordination of the breath and postures when practicing Tai Chi Chuan. If some boxing frames' routes are comparatively long and the air inhaled is not enough, a "small breath" can be added. Or the movement can be coordinated with two breaths. Never make yourself feel suffocated. In a word, be flexible, since lung's capacity is different for different people, the speed of practicing is various too, the route of hands and feet are also different, there is no doubt there will be difficulty in coordinating the breath and the movement. But one thing is certain that: try your best but not do it with difficulty to prevent from any accidental injury.

When starting practice the coordination of breath and boxing frames, you can consciously breathe deeply to lead the abdominal breathing method. After the breath can almost catch up with the boxing, you should weaken this consciousness and try to be oblivious of breathing.

The boxing breathing of Tai Chi Chuan is a perfect combination of breath and boxing under the guidance of consciousness. It is also a process connected the inside and the outside, which is important both for health benefit and martial arts. It is the required course of internal strength boxing. The practice of Tai Chi Chuan is divided into three progresses. The first is dropping the air to the public area, the second is to fulfill the body with the air and the third is consciousness, air and force. The most important part is the first one, which needs continuous practices and the core of it is the coordination of boxing postures and breath.

\section{References}

Xu, Guoqing \& Li, Xiaoqin. $(2005,4)$. On the relationship between consicouness and forces when practicing Tai Chi Chuan. Journal of Jiaozuo University.

Sun, Qingtian. (2009, 5). The three phases in teaching Tai Chi Chuan: physical form, breath, consciousness. Teaching of Physical Education.

Hou, Ting. (2007, 6). The breathing methods in practicing Tai Chi Chuan. Special Issue of Journal of Shanxi Normal University Sports Institute.

Li, Yongbin. (2009, 5). Analysis of single whip of Yang Tai Chi Chuan. Journal of Zhaoqing College. 